Vol. 5, No. 3-4, 2019

\author{
Oleksii Lanets ${ }^{1}$, Oleksandr Kachur ${ }^{2}$, Vitaliy Korendiy ${ }^{3}$ \\ ${ }^{1}$ Department of Mechanics and Automation Engineering, Lviv Polytechnic National University, Ukraine, Lviv, \\ S. Bandera Street 12, E-mail: poslanets1@gmail.com, ORCID 0000-0003-1053-8237 \\ ${ }^{2}$ Department of Mechanics and Automation Engineering, Lviv Polytechnic National University, Ukraine, Lviv, \\ S. Bandera Street 12, E-mail: dingof1992@gmail.com, ORCID 0000-0003-2263-6360 \\ ${ }^{3}$ Department of Mechanics and Automation Engineering, Lviv Polytechnic National University, Ukraine, Lviv, \\ S. Bandera Street 12, E-mail: vitaliy.nulp@gmail.com, ORCID 0000-0002-6025-3013
}

\title{
CLASSICAL APPROACH TO DETERMINING THE NATURAL FREQUENCY OF CONTINUAL SUBSYSTEM OF THREE-MASS INTER-RESONANT VIBRATORY MACHINE
}

Received: November 11, 2019 / Revised: December 20, 2019 / Accepted: December 28, 2019

(C) Lanets O., Kachur O., Korendiy V., 2019

\begin{abstract}
Problem statement. The three-mass vibratory system can be defined by five basic parameters: inertial parameters of the masses and stiffness parameters of two spring sets. Unlike the classical discrete system, the discrete-and-continual one consists of two rigid bodies connected by one spring set that form the discrete subsystem, and of the reactive mass considered as deformable (elastic) body characterized by certain stiffness and inertial parameters, which are related with one another. Purpose. The main objective of the paper consists in determining the first natural frequency of the continual subsystem of the three-mass discrete-and-continual vibratory machine. Methodology. While carrying out the investigations, it is used the classical theory of oscillations of straight elastic rods. Findings (results). The engineering technique of determining the first natural frequency of the continual subsystem of the three-mass vibratory machine is developed and approved by means of analytical calculations and numerical simulation. Originality (novelty). The optimal diagram of supporting the continual subsystem (elastic rod) is substantiated. The possibilities of exciting the vibrations of the three-mass discrete-and-continual mechanical system using the eccentric drive are considered. Practical value. The obtained research results and the developed calculation techniques can be used be engineers and designers dealing with various technological and manufacturing equipment that use vibratory drive. Scopes of further investigations. While carrying out further investigations, it is necessary to develop the model of combined discrete-and-continual system of three-mass vibratory machine, and to carry out the numerical simulation of the system's motion under different operational conditions.
\end{abstract}

Keywords: inertial parameters, stiffness, elastic rod, spring, numerical simulation, eccentric drive, calculation technique, operational conditions.

\section{Introduction}

The three-mass inter-resonant vibratory machines are considered as the most prospective from the viewpoint of the energy efficiency. The first designs of three-mass oscillatory systems can be found in the patents of the late $1930^{\text {th }}$ [1], [2]. In 1938, the inventor William W. Triggs published the patent, in which the necessity of adjusting the vibratory conveyer on the near-resonant operation mode was substantiated. The proposed oscillatory system emphasized on the advantages of multi-mass designs of vibratory equipment, which are related with better amplitude-frequency characteristics in inter-resonant operation modes. The possibility of moving the resonance peaks of the amplitude-frequency characteristic towards one another allows accumulating large dynamic potential of an oscillatory system in a narrow frequency band [3]. Due to this such oscillatory systems can be characterized by larger dynamic amplification factors 


\section{Oleksii Lanets, Oleksandr Kachur, Vitaliy Korendiy}

in comparison with the two-mass resonant systems. Because of such advantages, the three-mass interresonant oscillatory systems are usually called "highly effective" systems which can ensure larger amplitudes of oscillations of the masses under the same values of disturbing forces.

The further development of energy-efficient oscillatory systems can be carried out in the direction of reducing the values of the reactive mass and the stiffness of the corresponding spring set. Such requirements can be reached by means of implementation of a continual system in a form of an elastic body, which combines the inertial and rigidity parameters. The process of transformation of the reactive mass to the continual system is described in Fig. 1.

The proposed simplified diagram of the three-mass discrete-and-continual oscillatory system (Fig. 1) requires the estimation of the five basic parameters: inertial parameters of three masses and rigidity parameters of two spring sets. Unlike the discrete oscillatory system, the discrete-and-continual one consists of the elastic reactive body characterized by the distributed inertial and rigidity parameters, which depend on each other.
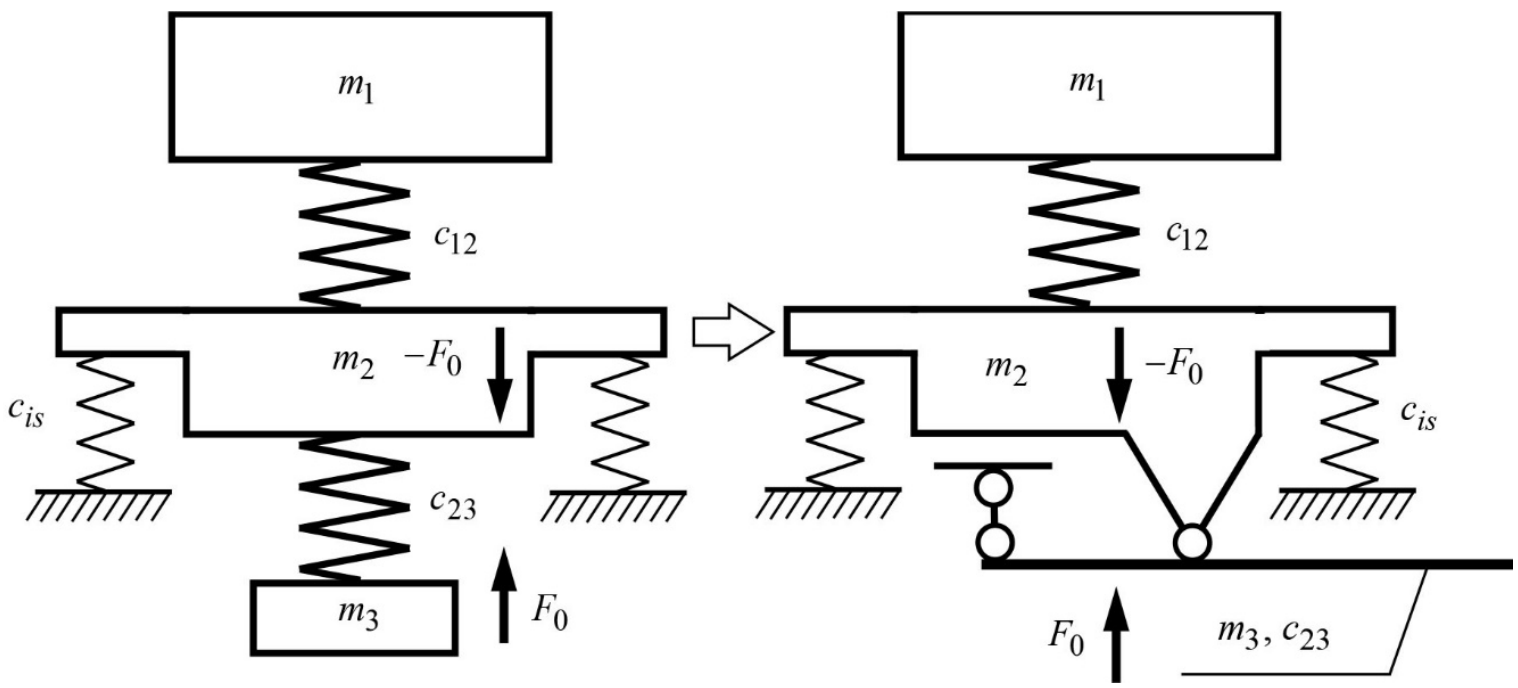

Fig. 1. Simplified diagrams of discrete and discrete-and-continual oscillatory systems of vibratory equipment

\section{Problem Statement}

The modern tendencies of industrial development require the creation of highly-efficient vibratory technological equipment able to implement the energy-saving technologies. One of possible ways of reaching the stated goal is to develop three-mass discrete-and-continual vibratory machines. The oscillatory systems of such machines must ensure the near-resonant operation mode, which can be reached by means of correct estimation of its inertial and rigidity parameters. The first natural frequency of a continual system (elastic rod) must be almost the same as the natural frequency of the discrete oscillatory system. The frequency band between these two frequencies is to be used as the major operation mode of a vibratory machine; this mode is called "inter-resonant" one. Therefore, the analytical determination of the first natural frequency of an elastic rod is an important step for further analysis of a continual system and its implementation in vibratory equipment, which will be characterized by larger dynamic amplification factor in comparison with two-mass resonant vibratory machines.

\section{Review of Modern Information Sources on the Subject of the Paper}

There exist a wide range of analytical techniques allowing the determination of natural frequencies of a rod. Among these techniques, it is necessary to mention the works of A. Buchacz [4], [5], [6], [7], who uses the theory of graphs. However, the graphs technique can be reduced to the classical theory of oscillations of straight elastic rods; this theory is described in [8], [9].

It is well-known that a straight rod has infinite number of forms of free oscillations, and each form of oscillations corresponds to a certain frequency value $\omega$, which is called a natural frequency. Its is 


\section{Classical Approach to Determining the Natural Frequency of Continual Subsystem of ...}

established that the first natural frequency of a rod is preferred to be used while designing and operating of various vibratory equipment. This frequency can be determined using the equation of free oscillations of a straight elastic rod taking into account the certain boundary conditions.

The differential equation of lateral (transverse) oscillations of a rod is following [8], [9]:

$$
E J_{z} \frac{\partial^{4} \eta(x, t)}{\partial x^{4}}+m_{r m} \frac{\partial^{2} \eta(x, t)}{\partial t^{2}}=0
$$

or

$$
c_{v}^{2} \frac{\partial^{4} \eta(x, t)}{\partial x^{4}}+\frac{\partial^{2} \eta(x, t)}{\partial t^{2}}=0
$$

where $c_{v}=\sqrt{\frac{E J_{z}}{m_{r m}}}$. is the speed of propagation of the deformation wave along the rod; $m_{r m}$ is the mass of a unit length of a rod (the mass of a running meter).

The solution of this equation can be found in the following form [8], [9]:

$$
\eta(x, t)=w(x) \sin \omega t .
$$

Substituting (3) into (1), the ordinary differential equation with respect to the deflection function $w(x)$ can be obtained:

$$
\frac{\mathrm{d}^{4} w(x)}{\mathrm{d} x^{4}}-\xi^{4} w(x)=0,
$$

in which there is adopted the following notation:

$$
\xi=\sqrt[4]{\frac{m_{r m} \omega^{2}}{E J_{z}}} .
$$

The equation (4) can be presented in the following form:

$$
E J_{z} w^{I V}(x)-\xi^{4} w(x)=0
$$

which have four independent partial solutions:

$$
\cos (\xi x), \sin (\xi x), \operatorname{ch}(\xi x), \operatorname{sh}(\xi x) .
$$

The general solution of the equation (6) is following:

$$
w(x)=A \cos (\xi x)+B \sin (\xi x)+C \operatorname{ch}(\xi x)+D \operatorname{sh}(\xi x),
$$

where $A, B, C, D$ are four arbitrary constants, which are chosen in such a way that the function (8) satisfies the boundary conditions, i.e. the conditions of fixation of the rod.

In some cases, it is suitable to seek a solution of the equation (6) in the following form:

$$
w(x)=A \cdot S(\xi x)+B \cdot T(\xi x)+C \cdot U(\xi x)+D \cdot V(\xi x),
$$

where $S(\xi x), T(\xi x), U(\xi x), V(\xi x)$ are combinations of circular and hyperbolic functions, which are called the Krylov functions [8], [9]:

$$
\begin{aligned}
& S(\xi x)=\frac{1}{2}(\operatorname{ch}(\xi x)+\cos (\xi x)) \\
& T(\xi x)=\frac{1}{2}(\operatorname{sh}(\xi x)+\sin (\xi x)) ; \\
& U(\xi x)=\frac{1}{2}(\operatorname{ch}(\xi x)-\cos (\xi x)) ; \\
& V(\xi x)=\frac{1}{2}(\operatorname{sh}(\xi x)-\sin (\xi x)) .
\end{aligned}
$$

The derivatives of these functions with respect to $x$ are following [9]:

$$
\begin{array}{lll}
S^{\prime}(\xi x)=\xi \mathrm{V}(\xi x) ; & S^{\prime \prime}(\xi x)=\xi^{2} U(\xi x) ; & S^{\prime \prime}(\xi x)=\xi^{3} T(\xi x) ; \\
T^{\prime}(\xi x)=\xi S(\xi x) ; & T^{\prime \prime}(\xi x)=\xi^{2} \mathrm{~V}(\xi x) ; & T^{\prime \prime}(\xi x)=\xi^{3} U(\xi x) ;
\end{array}
$$




\section{Oleksii Lanets, Oleksandr Kachur, Vitaliy Korendiy}

$$
\begin{array}{rlrl}
U^{\prime}(\xi x) & =\xi T(\xi x) ; & U^{\prime \prime}(\xi x)=\xi^{2} S(\xi x) ; & U^{\prime \prime \prime}(\xi x)=\xi^{3} \mathrm{~V}(\xi x) ; \\
V^{\prime}(\xi x)=\xi U(\xi x) ; & V^{\prime \prime}(\xi x)=\xi^{2} T(\xi x) ; & V^{\prime \prime \prime}(\xi x)=\xi^{3} S(\xi x) .
\end{array}
$$

In the case when $x=0$, the function (10), (11) take the following values [8]:

$$
\begin{array}{clll}
S(0)=1 ; & S^{\prime}(0)=0 ; & S^{\prime \prime}(0)=0 ; & S^{\prime \prime \prime}(0)=0 ; \\
T(0)=0 ; & T^{\prime}(0)=1 ; & T^{\prime \prime}(0)=0 ; & T^{\prime \prime \prime}(0)=0 ; \\
U(0)=0 ; & U^{\prime}(0)=0 ; & U^{\prime \prime}(0)=1 ; & U^{\prime \prime \prime}(0)=0 ; \\
V(0)=0 ; & V^{\prime}(0)=0 ; & V^{\prime \prime}(0)=0 ; & V^{\prime \prime \prime}(0)=1 .
\end{array}
$$

Using the approach presented above it is possible to determine the natural frequencies and the forms of free oscillations of a straight elastic rod.

\section{Objectives and Problems of Research}

The main purpose of the paper consists in substantiating the optimal diagram of fixation of a straight elastic rod used as a continual oscillatory system of a vibratory machine. As a conclusion of the carried out investigations its is necessary to analyse the possibilities of using the presented above technique for determining the exact values of natural frequencies of oscillations of a body with distributed inertial parameters, and to adjust this technique to the technique of calculation of discrete-and-continual oscillatory systems of vibratory equipment.

\section{Main Material Presentation}

Let us consider a rod (a body whose cross-section sizes are rather small in comparison with its length) as a continual oscillatory system of a vibratory machine. The calculation diagram of fixing the rod is presented in Fig. 2. The advantages of such fixation consists in formation of a cantilever able to be set into motion mechanically.

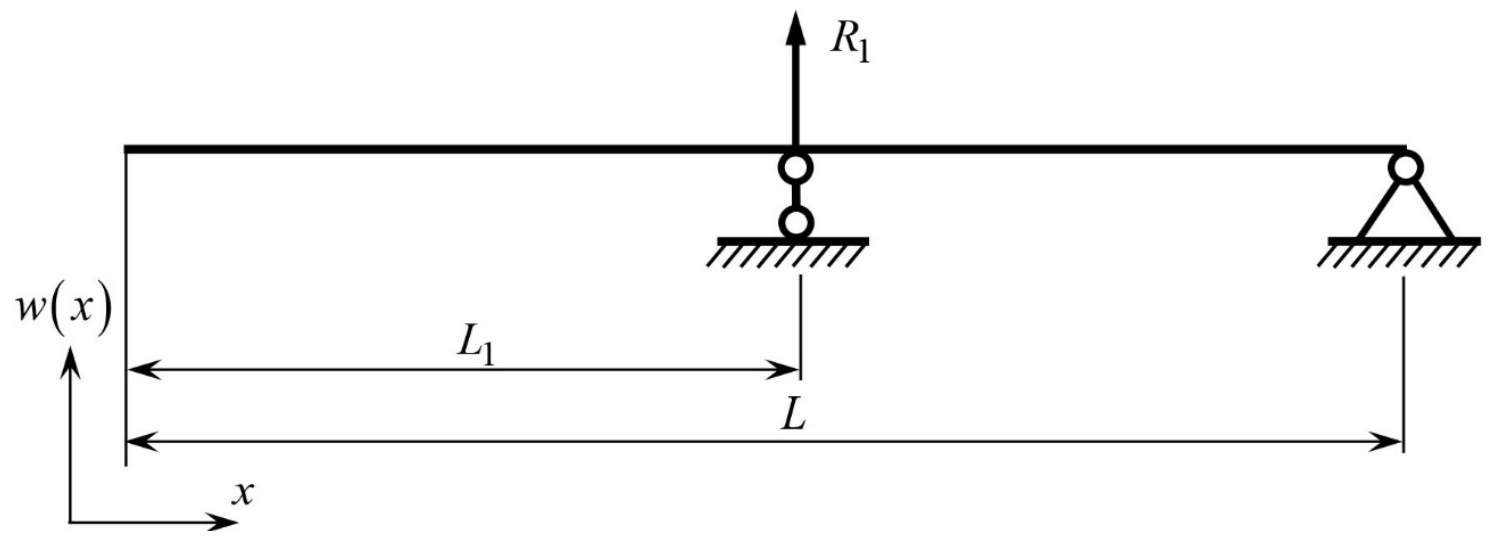

Fig. 2. Calculation diagram of the rod with the right hingedly supported end

The determination of a natural frequency of a continual system can be considered as a problem of analysing a straight elastic rod with several spans (Fig. 2). Two spans of the rod are analysed taking into account the reactions of supports. Using the material presented in [8], [9], the deflection equation of the right span (segment) of the rod can be presented as a function of deflection of the left span:

$$
w_{\text {right }}=w_{\text {left }}+\frac{R_{1}}{\xi^{3} E J_{z}} \cdot V\left(\xi\left(x-L_{1}\right)\right) \text {. }
$$

As a reference point it is taken the left end of the rod, which is not constrained. According to [8], the boundary conditions at the left end are following:

$$
\frac{\mathrm{d}^{2} w_{1}(x)}{\mathrm{d} x^{2}}=0
$$


Classical Approach to Determining the Natural Frequency of Continual Subsystem of ...

$$
\frac{\mathrm{d}^{3} w_{1}(x)}{\mathrm{d} x^{3}}=0
$$

Thus, taking into account (9) and (12), the general deflection equation of the left segment $\left(0 \leq x \leq L_{1}\right)$ of the rod is of the following form:

$$
w_{1}(x)=A \cdot S(\xi x)+B \cdot T(\xi x),
$$

where $\xi$ is to determined using the equation (5).

Using the expressions (13) and (16), let us write the general deflection equation of the right segment of the $\operatorname{rod}\left(L_{1} \leq x \leq L\right)$ :

$$
w_{1}(x)=A \cdot S(\xi x)+B \cdot T(\xi x)+\frac{R_{1}}{\xi^{3} E J_{z}} \cdot V\left(\xi\left(x-L_{1}\right)\right) .
$$

The equations (16) and (17) are written in the general form. In order to determine the natural frequency of the rod, let us deduce the corresponding frequency equation. Firstly, let us deduce a system of three equations, which satisfy the boundary conditions and the conditions in supports.

The first equation can be formed in the following way. The right end of the rod is hingedly supported, so the boundary conditions of its fixation are following [5]:

$$
\begin{gathered}
w_{1}(L)=0 ; \\
\frac{\mathrm{d}^{2} w_{1}(L)}{\mathrm{d} x^{2}}=0 .
\end{gathered}
$$

At the joining point of the right support $(x=L)$, according to the condition (18), the deflection is equal to zero. Using the equation (17), we obtain:

$$
w_{1}(L)=A \cdot S(\xi L)+B \cdot T(\xi L)+\frac{R_{1}}{\xi^{3} E J_{z}} \cdot V\left(\xi\left(L-L_{1}\right)\right)=0 .
$$

The second equation can be deduced on the basis of the condition (19), in which the second derivative of the deflection function $w_{r}(x)$ with respect to $x$ is equal to zero. Using the equation (17), for $x=L$, we obtain:

$$
\frac{\mathrm{d}^{2} w_{1}(L)}{\mathrm{d} x^{2}}=A \xi^{2} \cdot U(\xi L)+B \xi^{2} \cdot V(\xi L)+\frac{R_{1} \xi^{2}}{\xi^{3} E J_{z}} \cdot T\left(\xi\left(L-L_{1}\right)\right)=0 .
$$

Taking into account the fact that we have three unknowns: the constants $A, B$, and the reaction of the left support $R_{1}$, while the number of the derived equations is equal to two (equations (20) and (21)), it is necessary to deduce one more equation. It can be obtained using the condition of zero-deflection in the left support. Using (16), the third equation is following:

$$
w_{1}\left(L_{1}\right)=A \cdot S\left(\xi L_{1}\right)+B \cdot T\left(\xi L_{1}\right)=0 .
$$

Therefore, taking into account (20), (21), (22), the following system of three equations is obtained:

$$
\left\{\begin{array}{c}
A \cdot S(\xi L)+B \cdot T(\xi L)+\frac{R_{1}}{\xi^{3} E J_{z}} \cdot V\left(\xi\left(L-L_{1}\right)\right)=0 \\
A \cdot U(\xi L)+B \cdot V(\xi L)+\frac{R_{1}}{\xi^{3} E J_{z}} \cdot T\left(\xi\left(L-L_{1}\right)\right)=0 \\
A \cdot S\left(\xi L_{1}\right)+B \cdot T\left(\xi L_{1}\right)=0 .
\end{array}\right.
$$

Using the system of equations (23), let us form the frequency matrix:

$$
\Delta(\xi L)=\left|\begin{array}{ccc}
S(\xi L) & T(\xi L) & V\left(\xi\left(L-L_{1}\right)\right) \\
U(\xi L) & V(\xi L) & T\left(\xi\left(L-L_{1}\right)\right) \\
S\left(\xi L_{1}\right) & T\left(\xi L_{1}\right) & 0
\end{array}\right|
$$




\section{Oleksii Lanets, Oleksandr Kachur, Vitaliy Korendiy}

The determinant of the matrix (24) can be written as follows:

$$
\begin{gathered}
\Delta(\xi L)=-S(\xi L) \cdot T\left(\xi\left(L-L_{1}\right)\right) \cdot T\left(\xi L_{1}\right)+U(\xi L) \cdot V\left(\xi\left(L-L_{1}\right)\right) \cdot T\left(\xi L_{1}\right)+ \\
+S\left(\xi L_{1}\right) \cdot T(\xi L) \cdot T\left(\xi\left(L-L_{1}\right)\right)-S\left(\xi L_{1}\right) \cdot V\left(\xi\left(L-L_{1}\right)\right) \cdot V(\xi L),
\end{gathered}
$$

where

$$
\begin{gathered}
S(\xi L)=\frac{1}{2}(\operatorname{ch}(\xi L)+\cos (\xi L)) ; \\
T(\xi L)=\frac{1}{2}(\operatorname{sh}(\xi L)+\sin (\xi L)) ; \\
V\left(\xi\left(L-L_{1}\right)\right)=\frac{1}{2}\left(\operatorname{sh}\left(\xi\left(L-L_{1}\right)\right)-\sin \left(\xi\left(L-L_{1}\right)\right)\right) ; \\
U(\xi L)=\frac{1}{2}(\operatorname{ch}(\xi L)-\cos (\xi L)) ; \\
V(\xi L)=\frac{1}{2}(\operatorname{sh}(\xi L)-\sin (\xi L)) ; \\
T\left(\xi\left(L-L_{1}\right)\right)=\frac{1}{2}\left(\operatorname{sh}\left(\xi\left(L-L_{1}\right)\right)+\sin \left(\xi\left(L-L_{1}\right)\right)\right) ; \\
S\left(\xi L_{1}\right)=\frac{1}{2}\left(\operatorname{ch}\left(\xi L_{1}\right)+\cos \left(\xi L_{1}\right)\right) ; \\
T\left(\xi L_{1}\right)=\frac{1}{2}\left(\operatorname{sh}\left(\xi L_{1}\right)+\sin \left(\xi L_{1}\right)\right) .
\end{gathered}
$$

After substituting the expressions of the Krylov functions (26) into the equation (25), and after simplifying the obtained equation, we have:

$$
\begin{gathered}
\Delta(\xi L)=\frac{\operatorname{sh}(\xi L) \cdot \sin \left(\xi L-2 \xi L_{1}\right)}{8}-\frac{\operatorname{ch}(\xi L) \cdot \cos \left(\xi L-2 \xi L_{1}\right)}{8}+ \\
+\frac{\operatorname{sh}\left(\xi L-2 \xi L_{1}\right) \cdot \sin (\xi L)}{8}+\frac{\operatorname{ch}(\xi L) \cdot \cos (\xi L)}{8}+ \\
+\frac{\operatorname{sh}(\xi L) \cdot \sin (\xi L)}{4}+\frac{\operatorname{sh}\left(\xi L-\xi L_{1}\right) \cdot \sin \left(\xi L-\xi L_{1}\right)}{2}-\frac{\operatorname{sh}\left(\xi L_{1}\right) \cdot \operatorname{sh}\left(\xi L-\xi L_{1}\right) \cdot \cos (\xi L)}{4} .
\end{gathered}
$$

Using the applied software MathCad, let us graphically present the function $\Delta(\xi L)$ taking into account the following input data: the interval of calculation $\xi L \in[0 \ldots .3]$; the length of the rod $L=0.83 \mathrm{~m}$; the distance from the left end of the rod to the hinged support $L_{1}=0.475 \mathrm{~m}$. Based on the obtained results (Fig. 3), we can state that $\Delta(\xi L)=0$ when $\xi L \approx 2.7468$.

Before determining the cyclic frequency of free oscillations of the rod, let us evaluate the mass of the unit length of the rod (the mass of its running meter) $m_{r m}$ :

$$
m_{r m}=\rho h b_{n}=7820 \cdot 0.045 \cdot 5.8 \cdot 10^{-3}=1.694 \mathrm{~kg},
$$

where $\rho=7820 \mathrm{~kg} / \mathrm{m}^{3}$ is the specific mass (density) of the steel; $h=0.045 \mathrm{~m}$ is the width of the elastic rod; $b_{n}=5.8 \cdot 10^{-3} \mathrm{~m}$ is the thickness of the elastic rod.

Transforming the expression (5), we obtain the cyclic frequency of free oscillations of the rod:

$$
\omega_{f r}=(\xi L)^{2} \sqrt{\frac{E J_{z}}{m_{r m} L^{4}}}=2.768^{2} \sqrt{\left(1.96 \cdot 10^{11} \cdot \frac{0.045 \cdot\left(5.8 \cdot 10^{-3}\right)^{3}}{12}\right) /\left(1.694 \cdot 0.83^{4}\right)}=91.804 \frac{\mathrm{rad}}{\mathrm{s}} \text {, }
$$

where $E=1.96 \cdot 10^{11} \mathrm{~Pa}$ is the modulus of elongation (Young's modulus) of the steel; $J_{z}$ is the moment of inertia of the rod's cross-section about its neutral axis (for the rectangular cross-section $J_{z}=h b_{n}{ }^{3} / 12$ ). 


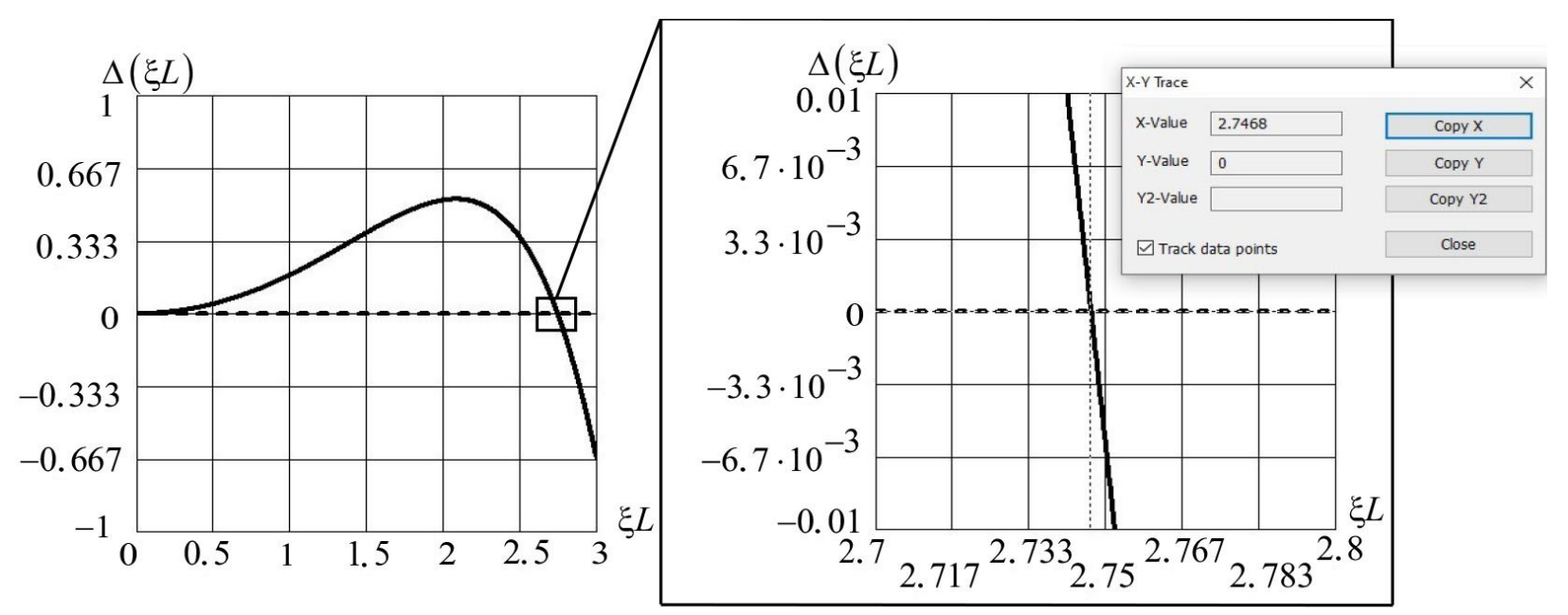

Fig. 3. The plot of the function $\Delta(\xi L)$ constructed on the interval $\xi L \in[0 \ldots 3]$

In order to verify the obtained results, let us carry out the analogous calculation for the case when the left end of the rod is hingedly supported, and the right end is not constrained (Fig. 4).

Let us write the boundary conditions for the left end of the rod [5]:

$$
\begin{gathered}
w_{2}(x)=0 ; \\
\frac{\mathrm{d}^{2} w_{2}(x)}{\mathrm{d} x^{2}}=0 .
\end{gathered}
$$

Taking into account the equations (9) and (12), the general deflection equation of the left segment of the $\operatorname{rod}\left(0 \leq x \leq L_{2}\right)$ takes the following form:

$$
w_{2}(x)=B \cdot T(\xi x)+D \cdot V(\xi x) .
$$

Using the expression (13) [8], [9], the general deflection equation of the right segment of the rod $\left(L_{2} \leq x \leq L\right)$ is following:

$$
w_{2}(x)=B \cdot T(\xi x)+D \cdot V(\xi x)+\frac{R_{2}}{\xi^{3} E J_{z}} \cdot V\left(\xi\left(x-L_{2}\right)\right) .
$$

The right end of the rod is free (unconstrained). Thus, the boundary conditions for the right end can be expressed as follows:

$$
\begin{aligned}
& \frac{\mathrm{d}^{2} w_{2}(L)}{\mathrm{d} x^{2}}=0 ; \\
& \frac{\mathrm{d}^{3} w_{2}(L)}{\mathrm{d} x^{3}}=0 .
\end{aligned}
$$

Using the equation (33), let us find the corresponding derivative of the function $w_{2}(x)$ with respect to $x$, and substitute the obtained expression into (34). Thus, for the case when $x=L$, we have:

$$
w_{2}(L)=B \cdot V(\xi L)+D \cdot T(\xi L)+\frac{R_{2}}{\xi^{3} E J_{z}} \cdot T\left(\xi\left(L-L_{2}\right)\right)=0 ;
$$

The second equation can be deduced using the second boundary condition (35). Using the equation (33), for the case when $x=L$, we have:

$$
\frac{\mathrm{d}^{2} w_{2}(L)}{\mathrm{d} x^{2}}=B \xi^{2} \cdot U(\xi L)+D \xi^{2} \cdot S(\xi L)+\frac{R_{2} \xi^{2}}{\xi^{3} E J_{z}} \cdot S\left(\xi\left(L-L_{2}\right)\right)=0 .
$$

Taking into account the fact that we have three unknowns: the constants $T, V$, and the reaction of the left support $R_{2}$, while the number of the derived equations is equal to two (equations (36) and (37)), it 


\section{Oleksii Lanets, Oleksandr Kachur, Vitaliy Korendiy}

is necessary to deduce one more equation. It can be obtained using the condition of zero-deflection in the right support. Using (32), the third equation is following:

$$
w_{2}\left(L_{2}\right)=B \cdot T\left(\xi L_{2}\right)+D \cdot V\left(\xi L_{2}\right)=0 .
$$

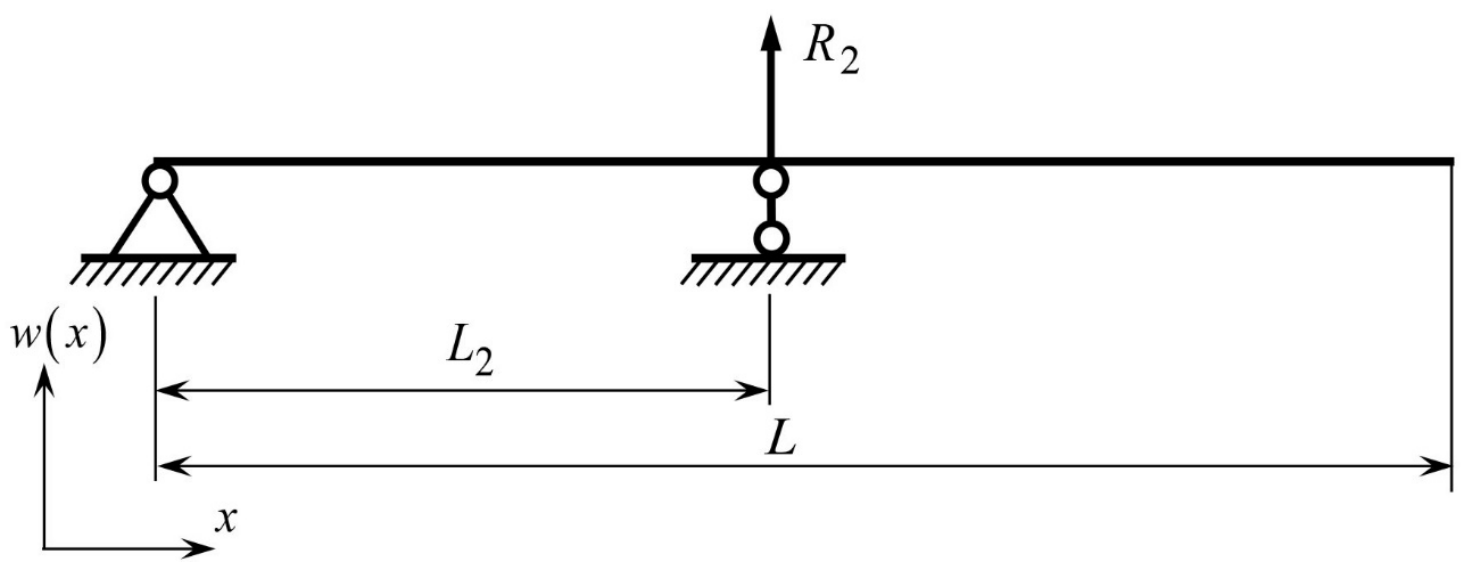

Fig. 4. Calculation diagram of the rod with the left hingedly supported end

Taking into account (36), (37), (38), the system of three equations is following:

$$
\left\{\begin{array}{c}
B \cdot V(\xi L)+D \cdot T(\xi L)+\frac{R_{2}}{\xi^{3} E J_{z}} \cdot T\left(\xi\left(L-L_{2}\right)\right)=0 ; \\
B \cdot U(\xi L)+D \cdot S(\xi L)+\frac{R_{2}}{\xi^{3} E J_{z}} \cdot S\left(\xi\left(L-L_{2}\right)\right)=0 ; \\
B \cdot T\left(\xi L_{2}\right)+D \cdot V\left(\xi L_{2}\right)=0 .
\end{array}\right.
$$

The frequency matrix of the system (39) takes the following form:

$$
\Delta(\xi L)=\left|\begin{array}{ccc}
V(\xi L) & T(\xi L) & T\left(\xi\left(L-L_{2}\right)\right) \\
U(\xi L) & S(\xi L) & S\left(\xi\left(L-L_{2}\right)\right) \\
T\left(\xi L_{2}\right) & V\left(\xi L_{2}\right) & 0
\end{array}\right| .
$$

The determinant of the frequency matrix (40) equals:

$$
\begin{gathered}
\Delta(\xi L)=-V(\xi L) \cdot S\left(\xi\left(L-L_{2}\right)\right) \cdot V\left(\xi L_{2}\right)+U(\xi L) \cdot T\left(\xi\left(L-L_{2}\right)\right) \cdot V\left(\xi L_{2}\right)+ \\
\quad+T\left(\xi L_{2}\right) \cdot T(\xi L) \cdot S\left(\xi\left(L-L_{2}\right)\right)-T\left(\xi L_{2}\right) \cdot T\left(\xi\left(L-L_{2}\right)\right) \cdot S(\xi L),
\end{gathered}
$$

where

$$
\begin{gathered}
V(\xi L)=\frac{1}{2}(\operatorname{sh}(\xi L)-\sin (\xi L)) ; \\
T(\xi L)=\frac{1}{2}(\operatorname{sh}(\xi L)+\sin (\xi L)) ; \\
T\left(\xi\left(L-L_{2}\right)\right)=\frac{1}{2}\left(\operatorname{sh}\left(\xi\left(L-L_{2}\right)\right)+\sin \left(\xi\left(L-L_{2}\right)\right)\right) ; \\
U(\xi L)=\frac{1}{2}(\operatorname{ch}(\xi L)-\cos (\xi L)) ; \\
S(\xi L)=\frac{1}{2}(\operatorname{ch}(\xi L)+\cos (\xi L)) ; \\
S\left(\xi\left(L-L_{2}\right)\right)=\frac{1}{2}\left(\operatorname{sh}\left(\xi\left(L-L_{2}\right)\right)-\sin \left(\xi\left(L-L_{2}\right)\right)\right) ; \\
T\left(\xi L_{2}\right)=\frac{1}{2}\left(\operatorname{sh}\left(\xi L_{2}\right)+\sin \left(\xi L_{2}\right)\right) ;
\end{gathered}
$$




$$
V\left(\xi L_{2}\right)=\frac{1}{2}\left(\operatorname{sh}\left(\xi L_{2}\right)-\sin \left(\xi L_{2}\right)\right)
$$

After substituting the expressions of the Krylov functions (42) into the equation (41), and after simplifying the obtained equation, we have:

$$
\begin{gathered}
\Delta(\xi L)=\frac{\operatorname{ch}(\xi L) \cdot \cos (\xi L)}{8}-\frac{\operatorname{sh}(\xi L) \cdot \sin \left(\xi L-2 \xi L_{2}\right)}{8}- \\
-\frac{\operatorname{sh}\left(\xi L-2 \xi L_{2}\right) \cdot \sin (\xi L)}{8}-\frac{\operatorname{ch}(\xi L) \cdot \cos \left(\xi L-2 \xi L_{2}\right)}{8}+ \\
+\frac{\operatorname{sh}(\xi L) \cdot \sin (\xi L)}{4}+\frac{\operatorname{sh}\left(\xi L_{2}\right) \cdot \sin \left(\xi L_{2}\right)}{2}-\frac{\operatorname{sh}\left(\xi L_{2}\right) \cdot \operatorname{sh}\left(\xi L-\xi L_{2}\right) \cdot \cos (\xi L)}{4} .
\end{gathered}
$$

Using the applied software MathCad, let us graphically present the function $\Delta(\xi L)$ taking into account the previously mentioned input data: $\xi L \in[0 \ldots 3] ; L=0.83 \mathrm{~m} ; L_{1}=0.475 \mathrm{~m}$. Based on the obtained results (Fig. 5), we can state that $\Delta(\xi L)=0$ when $\xi L \approx 2.7468$. This means that the results of both calculations carried out for two different diagrams of fixation of the rod (Figs. 2 and 4) are in agreement with one another (see Figs. 3 and 5). Thus, we can conclude that the calculation of the first natural frequency of the rod is carried out correctly.

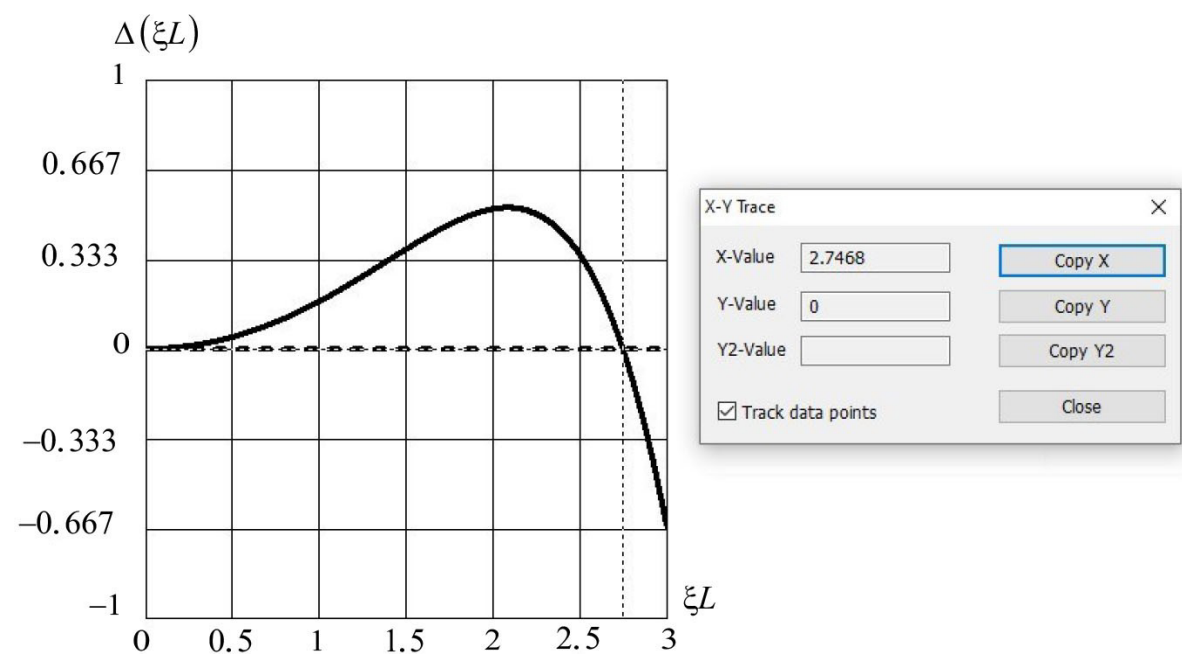

Fig. 5. The plot of the function $\Delta(\xi L)$ constructed on the interval $\xi L \in[0 \ldots 3]$

Let us substantiate the correctness of the analytical calculations by means of frequency analysis of the rod in SolidWorks Simulation software [10]. The frequency analysis of the solid model of the rod is performed using the finite-element method. Before carrying out the simulation, it is necessary to apply the correct constraints upon the body, which correspond to the calculation diagram presented in Fig. 2.

The modelling of natural frequencies and forms of free oscillations is performed taking into account the following assumptions: damping, friction and external forces are neglected [10]. Let us design a solid model of the elastic rod (Fig. 6) using the following dimensions: $h=0.045 \mathrm{~m}$ is the width of the rod's cross-section; $b_{n}=5.8 \cdot 10^{-3} \mathrm{~m}$ is the thickness of the rod (the height of the rod's cross-section); $L=0.83 \mathrm{~m}$ is the length of the rod; $L_{1}=0.475 \mathrm{~m}$ is the distance from the free end of the rod to the hinged support. The physical properties of the rod's material are following: $\rho=7820 \mathrm{~kg} / \mathrm{m}^{3}$ is the specific mass (density) of steel; $E=1.96 \cdot 10^{11} \mathrm{~Pa}$ is the modulus of elongation (Young's modulus) of the steel.

Let us determine the cyclic natural frequency of the rod in accordance with the obtained results of simulation (see Fig. 6):

$$
\omega_{f r s}=v_{f r s} 2 \pi=14.648 \cdot 2 \pi=92.036 \mathrm{rad} / \mathrm{s} .
$$




\section{Oleksii Lanets, Oleksandr Kachur, Vitaliy Korendiy}

The natural frequency value obtained by simulation in SolidWorks Simulation software is almost equal to the one analytically calculated by the equation (29). This means that the proposed analytical approach to determining the first natural frequency of the straight elastic rod can be effectively used while calculating the rigidity (stiffness) parameters of continual oscillatory systems of vibratory equipment.

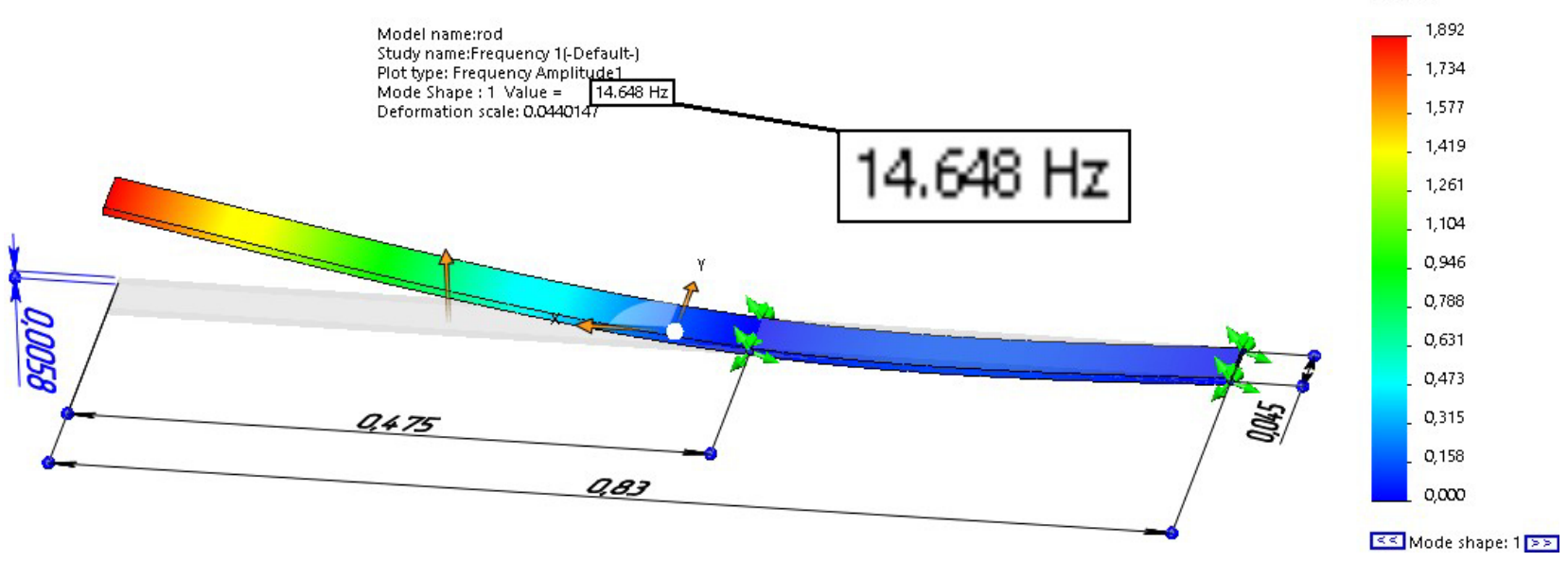

Fig. 6. The frequency diagram of the straight elastic rod obtained with the help of the finite-element method in SolidWorks Simulation software

\section{Conclusions}

It is established that one of the optimal diagrams of fixation of a continual oscillatory system presented by a straight elastic rod consists of two hinged supports (Fig. 2), one of which can be disturbed by means of an eccentric drive. The main advantage of such a fixation consists in the fact that the rod forms a cantilever with improved inertia parameters (relative to its length); and the cantilever can be set in motion mechanically through the supports.

It is proved that the proposed approach to determining the natural frequencies of oscillations of the straight elastic rod does not depend on the location of the hinged supports (left or right end of the rod, see Figs. 2 and 4). The only difference consists in the form of the boundary conditions, while the reference point is placed at the left end of the rod.

The proposed analytical approach to determining the first natural frequency of the straight elastic rod can be effectively used while calculating the rigidity (stiffness) parameters of continual oscillatory systems of vibratory equipment.

The basic peculiarities of forming the finite-element model of the continual oscillatory system in SolidWorks Simulation software are considered. The finite-element method is used for frequency analysis of the straight elastic rod. The results of computer simulation match the results of analytical calculation with a high degree of accuracy. This proves the correctness of the proposed calculation technique.

\section{References}

[1] W. W. Triggs, "Improvements in and relating to method of and apparatus for conveying and conditioning materials", GB494206A, October 21, 1938.

[2] O. S. Lanets, "Rozvytok mizhrezonansnykh mashyn z elektromahnitnym pryvodom" ["Development of inter-resonant machines with electromagnetic drive"], Avtomatyzatsiya vyrobnychykh protsesiv u mashynobuduvanni ta pryladobuduvanni [Industrial Process Automation in Engineering and Instrumentation], vol. 42, pp. 3-18, 2008. [in Ukrainian].

[3] O. Lanets, Osnovy rozrakhunku ta konstruiuvannia vibratsiinykh mashyn [Fundamentals of Analysis and Design of Vibratory Machines], Lviv, Ukraine: Lviv Polytechnic Publishing House, 2018. [in Ukrainian].

[4] A. Buchacz, "Characteristics of discrete-continuous flexibly vibrating mechatronic system", Journal of Achievements in Materials and Manufacturing Engineering, vol. 28, issue 1, pp. 43-46, May 2008.

[5] A. Buchacz, "Dynamical flexibility of discrete-continuous vibrating mechatronic system", Journal of Achievements in Materials and Manufacturing Engineering, vol. 28, issue 2, pp. 159-166, June 2008. 


\section{Classical Approach to Determining the Natural Frequency of Continual Subsystem of ...}

[6] A. Buchacz, "Calculation of flexibility of vibrating beam as the subsystem of mechatronic system by means the exact and approximate methods", Proceedings in Applied Mathematics and Mechanics, vol. 9, issue 1, pp. 373-374, 2009.

[7] A. Buchacz, "The supply of formal notions to synthesis of the vibrating discrete-continuous mechatronic systems", Journal of Achievements in Materials and Manufacturing Engineering, vol. 44, issue 2, pp. 168-178, 2011.

[8] I. M. Babakov, Teoriya kolebaniy [Theory of oscillations]. Leningrad, Russia: Nauka Publ., 1968. [in Russian].

[9] S. D. Ponomarev et al., Raschety na prochnost $v$ mashinostroyenii [Strength calculations in mechanical engineering]. Moscow, Russia: Mashgiz Publ., 1959. [in Russian].

[10] P. M. Kurowski, Vibration Analysis with SOLIDWORKS Simulation 2018. Mission, KS, USA: SDC Publications, 2018. 